\title{
Endodontia em sessão única ou múltipla: revisão da literatura
}

\section{Endodontics in single or multiple visits: literature review}

\author{
Marcos Sergio Endo* \\ Ana Clara Lobianco dos Santos ${ }^{* *}$ \\ Angelo Jose Pavan*** \\ Alfredo Franco Queiroz ${ }^{* * *}$ \\ Nair Narumi Orita Pavan ${ }^{* * * * *}$
}

\section{Resumo}

Atualmente, com os avanços tecnológicos e o aperfeiçoamento do preparo químico-mecânico durante a limpeza e modelagem do canal radicular, o tempo para concretização desse procedimento foi reduzido, possibilitando a realização do tratamento em sessão única. Objetivo: discutir e confrontar, a partir de evidências científicas, os achados sobre dor pós-operatória e taxa de reparação em tratamentos endodônticos realizados em sessão única ou múltipla. Métodos: essa revisão da literatura baseou-se na pesquisa de trabalhos nas bases de dados PubMed, Science Direct, Scopus, Scielo e Medline. Revisão da literatura: o tratamento endodôntico em sessão única pode ser realizado nos casos diagnosticados como pulpite irreversível. No entanto, em casos de necrose pulpar, com ou sem periodontite apical, a literatura é controversa e as opiniões variam quanto aos riscos e benefícios da sessão única e da múltipla. Aliando-se a outras vantagens, como economia de tempo, custo-benefício, melhor aceitação do paciente e redução dos riscos de infecção entre as sessões, a sessão única pode ser indicada, com exceção de alguns casos, como na presença de exsudato. Considerações finais: o tratamento em sessão única e em sessão múltipla mostrou resultados semelhantes considerando a taxa de reparação e a dor pós-operatória. Assim, a tomada de decisão clínica em optar por um tratamento endodôntico em sessão única ou múltipla deve ser baseada em evidências clínico-científicas.

Palavras-chave: Endodontia. Dor pós-operatória. Tratamento.

\section{Introdução}

O tratamento endodôntico é realizado, na maioria dos dentes, com taxas de sucesso variáveis ${ }^{1,2}$. Grande parte dos dentistas realiza esse tratamento em sessões múltiplas ${ }^{3}$. No entanto, nas últimas décadas, mais de 70\% das escolas, em diversas áreas geográficas, e pesquisadores defendem o tratamento em sessão única ${ }^{4}$.

É pouco contestada a possibilidade de realização do tratamento do canal radicular em sessão única nos casos diagnosticados como pulpite irreversível $^{4,5}$. No entanto, em casos de necrose pulpar com ou sem periodontite apical, a literatura é controver$\mathrm{sa}^{6}$. A periodontite apical é causada por bactérias no interior dos canais, e o seu tratamento deve ser feito com a remoção da causa. O preparo mecânico associado a uma irrigação pode promover culturas bacterianas negativas em $40 \%$ a $60 \%$ dos dentes tratados $^{7,8}$. Além disso, o uso da medicação intracanal foi sugerido para potencializar a obtenção de culturas negativas ${ }^{9}$.

Historicamente, inúmeros tratamentos e medicações entre sessões foram utilizados para a terapia de dentes infeccionados, mas ao longo dos anos o número de sessões foi reduzido. Para os casos de sessões múltiplas, um curativo de demora à base de hidróxido de cálcio tem sido proposto como padrão-ouro ${ }^{4}$. Assim, acreditava-se que a taxa de reparação do tratamento em sessões múltiplas devia ser mais elevado do que o tratamento em sessão única ${ }^{9}$.

\footnotetext{
Doutor em Endodontia, professor adjunto, Departamento de Odontologia, Área de Endodontia, Universidade Estadual de Maringá, Maringá, PR, Brasil. Especialista em Endodontia, Departamento de Odontologia, Área de Endodontia, Universidade Estadual de Maringá, Maringá, PR, Brasil.

Doutor, professor adjunto, Departamento de Odontologia, Universidade Estadual de Maringá, Maringá, PR, Brasil.

*** Mestre em Endodontia, professor assistente, Departamento de Odontologia, Área de Endodontia, Universidade Estadual de Maringá, Maringá, PR, Brasil.

***** Doutora, professora adjunta, Departamento de Odontologia, Área de Endodontia, Universidade Estadual de Maringá, Maringá, PR, Brasil.
} 
O tratamento endodôntico em sessão única ou múltipla tem sido objeto de discussão de longa data na comunidade endodôntica. De fato, a tentativa de concluir esse tratamento em sessão única é documentada desde o final do século $\mathrm{XIX}^{10}$, embora ainda não se tenha obtido uma conclusão definitiva. Logo após, verificam-se relatos sobre a obturação imediata, descrevendo os critérios para o sucesso com base na limpeza e modelagem do canal radicular, assim como nos métodos de remoção do conteúdo infeccioso $^{11}$. As técnicas utilizadas antigamente eram primitivas e mostravam baixa taxa de sucesso para os tratamentos realizados em sessão única. A terapia endodôntica geralmente era realizada em sessões múltiplas, e exigia um tempo considerável para a finalização do tratamento ${ }^{12}$.

O uso de técnicas e equipamentos modernos, como magnificação (aumento da imagem), localizadores eletrônicos foraminais e aparelhos mecanizados utilizando limas de NiTi e sistemas reciprocantes, fizeram com que se reduzisse o tempo para concretização desse tratamento, permitindo sua conclusão em uma única sessão.

Atualmente, verifica-se muito interesse dos profissionais em concluir o tratamento em uma única sessão. Sendo assim, este trabalho de revisão da literatura teve como objetivo discutir e confrontar, a partir de evidências científicas, os achados sobre dor pós-operatória e taxa de reparação após o tratamento endodôntico em sessão única e múltipla.

\section{Metodologia}

Esta revisão da literatura foi elaborada com base na pesquisa de trabalhos nas bases de dados PubMed, Science Direct, Scopus, Scielo e Medline. Os descritores utilizados foram: single visit root canal treatment, multiple visit root canal treatment, postoperative pain, post-obturation pain, success, treatment outcome.

\section{Revisão de literatura}

O raciocínio biológico fundamental para alcançar o sucesso do tratamento endodôntico consiste basicamente em eliminar micro-organismos do sistema de canais radiculares, criando um ambiente favorável para a reparação. Porém, dificilmente se consegue um ambiente livre de bactérias, mesmo após adequada limpeza e modelagem dos canais radiculares ${ }^{8}$. Duas abordagens foram propostas para resolver esse problema. Em uma abordagem, as bactérias residuais são eliminadas ou impedidas de recolonizar os canais radiculares por meio da utilização de uma medicação intracanal. Em seu estudo, Orstavik et al. ${ }^{13}$ (1991) verificaram que o hidróxido de cálcio não permitiu a eliminação completa dos micro-organismos, além disso, observaram que houve a recolonização bacteriana após o seu uso.
No entanto, mesmo uma cultura negativa antes da obturação dos canais não nos fornece garantia de reparação em todos os casos ${ }^{14,15}$. A outra abordagem consiste na eliminação das bactérias remanescentes ou em torná-las inofensivas por sepultamento após uma obturação hermética tridimensional, para privar a nutrição dos micro-organismos e o espaço necessário para sobreviver e se multiplicar ${ }^{15,16}$.

Contudo, nem o tratamento em sessão única nem o de sessão múltipla podem eliminar completamente as colônias microbianas ${ }^{17}$. Em relação à redução de endotoxinas, o tratamento em sessão única mostrou-se menos eficaz do que em sessões múltiplas ${ }^{18}$.

Os fatores a serem considerados na escolha da modalidade de tratamento endodôntico são a habilidade do operador, a experiência clínica, as condições do dente (dente vital ou não vital, sintomático ou assintomático, presença ou ausência de exsudato e edema), o adequado tempo de tratamento, as limitações de tempo do paciente, a história médica e as considerações anatômicas e biológicas.

As vantagens em realizar o tratamento em sessão única são: a) redução no número de idas ao consultório; b) redução de infiltração e/ou contaminação entre as sessões; c) redução dos custos em relação ao tempo clínico; d) evita repetidas anestesias e a colocação do isolamento absoluto; e) minimiza possíveis iatrogenias (perfuração, desvio, rasgo, e extrusão de debris/irrigantes devido a exposições mais longas durante a instrumentação); f) permite que os dentistas realizem a obturação, já que o profissional está familiarizado com a anatomia do canal radicular; g) permite a retomada da função do dente de maneira eficiente e imediata após o tratamento.

O principal objetivo ao realizar o tratamento em sessão única é a obtenção de sucesso em termos de prevenção e, quando necessário, a reparação da periodontite apical, minimizando qualquer desconforto dos pacientes ${ }^{19}$. No entanto, essa opção de tratamento apresenta algumas desvantagens. A conclusão em sessão única pode envolver restrições de tempo e causar fadiga, tanto do clínico quanto do paciente. A disfunção da articulação temporomandibular pré-existente é uma contraindicação, pois o tempo longo de tratamento pode induzir o paciente ao estresse. Além disso, o tratamento em sessão única não deve ser realizado quando os canais radiculares não estão secos devido ao exsudato originário da periodontite apical aguda ${ }^{20}$.

Tradicionalmente, o tratamento endodôntico é realizado em várias sessões, utilizando uma medicação intracanal entre o preparo químico-mecânico e a obturação, que visa, principalmente, reduzir ou eliminar micro-organismos e seus subprodutos do sistema de canais radiculares antes da obturação. $\mathrm{O}$ tratamento em sessões múltiplas é bem aceito e seguro $^{21}$; no entanto, nos últimos anos, não foram observadas diferenças significantes quanto à redu- 
ção de micro-organismos nos casos tratados em sessão única e múltipla ${ }^{22}$.

Ademais, com o uso dos sistemas rotatórios e reciprocantes e o aperfeiçoamento técnico do preparo químico-mecânico durante a desinfecção do canal radicular, o tratamento em uma única sessão passou a ser mais conveniente. Aliado a outras vantagens, incluindo economia de tempo, custo-benefício, melhor aceitação do paciente e redução dos riscos de infecção entre as sessões, o tratamento em sessão única tornou-se um protocolo aceitável ${ }^{23}$.

O tratamento do canal radicular em sessão única é uma prática comum e oferece vantagens, tais como uma taxa de flare-up reduzida ${ }^{24}$ e considerações clínicas de planejamento. Um estudo relatou que quase $70 \%$ dos endodontistas tratariam dentes com necrose pulpar e abscesso apical crônico em sessão única ${ }^{25}$. Outra pesquisa mostrou que cerca de $70 \%$ das instituições de ensino de graduação nos Estados Unidos da América (EUA) também incentivam o tratamento em sessão única ${ }^{26}$.

No entanto, o fato de esse tipo de tratamento ser amplamente adotado não indica que essa prática é biologicamente a mais apropriada. $\mathrm{O}$ argumento para a sessão única depende de conveniência, questão biológica do referido caso, aceitação do paciente e redução da dor pós-operatória. A questão é controversa e as opiniões variam quanto aos riscos e benefícios relativos dos tratamentos endodônticos realizados em sessão única ou múltipla.

O conceito subjacente, como descrito por Oliet ${ }^{16}$ (1983), é que não há diferença nos critérios de tratamento para assegurar um resultado bem-sucedido entre o tratamento em sessão única e múltipla. Os critérios incluem diagnóstico correto e preciso, adequada seleção de casos e uso de técnicas endodônticas contemporâneas.

Carrotte $^{27}$ (2005) propôs que seria adequado prosseguir com a obturação uma vez que o dentista tenha concluído o preparo do canal radicular e o desbridamento. Se os canais não estiverem secos devido à presença de exsudatos apicais persistentes, deve-se inserir a medicação intracanal. Uma restauração temporária com um bom selamento coronário é necessária para impedir infiltrações. Caso contrário, haverá recolonização bacteriana, o que prejudica o sucesso da endodontia ${ }^{4}$.

\section{Dor pós-operatória e reparação periapical: sessão única versus sessões múlitiplas}

A dor pós-operatória é definida como a dor de qualquer grau que ocorre após o início do tratamento, enquanto flare-up, quando o início ou continuação da dor e/ou edema após o tratamento é de tal gravidade que perturba a qualidade de vida do paciente o suficiente para que ele procure atendimen- to sem hora marcada. Flare-up é raro, apresentando uma média de $3 \% 4$.

O desconforto é a principal complicação em curto prazo após o tratamento endodôntico. Infelizmente, a mensuração de desconforto oferece abertura para erros, por ser de caráter subjetivo. Por isso é necessário avaliar o nível de desconforto em categorias com antecedência. Yoldas et al. ${ }^{28}$ (2004) tiveram a preocupação em categorizar a dor dos pacientes. $\mathrm{O}$ desconforto após o tratamento pode ser classificado em curto, médio e longo prazo. Em comparação com a abordagem em sessão múltipla, a prevalência de dor pós-obturação em curto prazo foi significativamente menor em sessão única ${ }^{29}$.

A seleção de procedimentos clínicos na terapia endodôntica depende não apenas de sua eficácia ou consequências biológicas, mas também da minimização do desconforto dos pacientes. Nas últimas décadas, a discussão sobre o tratamento em sessão única ou múltipla ganhou atenção; no entanto, nenhum consenso foi obtido ${ }^{4}$. Dor e edema são, muitas vezes, indicadores de um processo de doença associada a um dente acometido. A endodontia destina-se a reverter o processo da doença e, assim, a eliminar os sinais e sintomas associados. Quando surgir dor e/ou edema após o tratamento, o resultado pode ser muito aflitivo para o paciente e para o operador.

Os pacientes podem considerar a dor pós-operatória e flare-up como uma referência contra a qual são testadas as habilidades do profissional. Mesmo que a dor pós-operatória não seja um adequado método para se avaliar o sucesso do tratamento por ser de caráter transitório, ela tem sido amplamente utilizada como argumento a favor ou contra em se tratando de sessão única. Houve um período, em que a maioria dos endodontistas nos EUA acreditava que haveria mais dor se o tratamento fosse realizado em sessão única ${ }^{30}$.

Os estudos sobre tratamento endodôntico em sessão única têm direcionado maior atenção à dor pós-operatória e aos flare-ups, apesar de ter-se demonstrado que a dor não tem efeito sobre o sucesso em longo prazo da reparação periapical ${ }^{31}$. A dor pós-obturação pode estar relacionada a diferentes fatores, incluindo infecção, retratamento, dor pré-operatória, medicação intracanal, agressões físicas e químicas aos tecidos periapicais ${ }^{28}$.

É reconhecido que uma abordagem em sessão única também pode prevenir a ocorrência de dor devido à reinfecção dos canais como consequência da invasão bacteriana a partir de uma restauração temporária ${ }^{4}$. Já as sessões múltiplas, ao contrário, envolvem a inserção de um selamento temporário e uma contínua estimulação física e química sobre os tecidos periapicais. A recontaminação dos canais radiculares ou invasão microbiana secundária pode ser outra razão que leva à maior dor pós-obturação no grupo de sessões múltiplas ${ }^{29}$. 
Estudos demonstram que a incidência de dor pós-obturação diminuiu ao longo do tempo, e que foi maior durante as primeiras 24-48 horas após o procedimento, com uma redução constante nos sete dias seguintes ${ }^{28,29}$. Em termos de período pós-obturação em médio prazo, a incidência de dor, tanto em sessão única quanto múltipla, foi reduzida, e a diferença entre os dois procedimentos não foi significativa.

Apenas um estudo ${ }^{5}$ investigando complicação tardia (um mês) pós-obturação foi identificado. Nesse estudo prospectivo, foi observada a incidência significativamente mais elevada $(p<0,01)$ de dor pós-obturação nos casos realizados em sessão múltipla (38\%), quando comparado à sessão única (27\%), após 24 horas. No final do período de observação, todos os pacientes apresentaram-se assintomáticos ${ }^{5}$.

$\mathrm{Na}$ revisão sistemática conduzida por Figini et al. ${ }^{19}$ (2008), relatou-se não haver diferença na incidência de dor pós-operatória em curto prazo entre os dois regimes de tratamento. Isso pode ser explicado pelas diferenças de participantes e intervenções clínicas. Nessa revisão ${ }^{19}$, os dentes com polpas vitais e não vitais foram avaliados, enquanto que no estudo de Su et al..$^{29}$ (2011) foram incluídos apenas os casos de polpas necróticas.

Segundo Wong et al. ${ }^{20}$ (2014), há relatos de que dor pós-operatória ou complicações foram comumente detectadas após procedimentos endodônticos, com duração que varia desde o mesmo dia até semanas. As complicações incluem edema, dor à percussão, mobilidade e perturbação sistêmica. A dor foi relatada como sendo de grau leve a formas graves, descritas como flare-ups. Além disso, a ausência de dor pós-operatória não pôde ser garantida em nenhuma das duas modalidades de tratamento.

De acordo com uma revisão sistemática e meta-análise realizada por Sathorn et al. ${ }^{32}$ (2005), foram identificados somente três estudos clínicos que preencheram os padrões mais altos em relação ao nível de evidência ${ }^{14,15,33}$. Os autores concluíram que não houve diferença estatisticamente significante na reparação entre os tratamentos endodônticos realizados em sessão única e múltipla.

Wong et al. ${ }^{20}$ (2014) afirmaram em sua revisão sistemática, por meio de uma meta-análise, que as complicações pós-operatórias apresentaram-se similares tanto no tratamento endodôntico realizado em sessão única quanto em múltiplas sessões. Assim, o número de sessões não interferiu no processo de reparo ou na taxa de sucesso.

Percebe-se que devem ser realizados mais estudos com um maior número amostral para que seja possível desenvolver uma análise estatística confiável. Além disso, um ensaio clínico prospectivo randomizado seria recomendável para comprovar a taxa de dor pós-operatória e de sucesso do tratamento em sessão única e em múltiplas sessões.

El Mubarak et al. ${ }^{3}$ (2010) observaram que a incidência de dor pós-operatória associada a den- tes previamente sintomáticos foi de $15,9 \%$, em comparação a $7,1 \%$ para os dentes assintomáticos ( $p<0,05)$. Esse achado está de acordo com o apresentado por outros estudos ${ }^{24,34}$.

O tratamento endodôntico envolve várias etapas críticas destinadas à redução do conteúdo infeccioso. Micro-organismos podem ser extruídos para os tecidos perirradiculares ou mantidos no interior do canal, o que pode ser responsável pela inflamação e pelos sintomas pós-operatórios. El Mubarak et al. ${ }^{3}$ (2010) detectaram que a dor pós-operatória apresentou correlação positiva com dentes não vitais $(13,7 \%)$ ao se comparar com dentes vitais $(5,4 \%)$; corroborando com os achados de Albashaireh e Alnegrish $^{5}$ (1998).

Há conflito de opiniões no que diz respeito à influência do número de sessões na execução do tratamento endodôntico e à dor pós-operatória. Pesquisadores encontraram que a incidência de dor pós-operatória em sessões múltiplas foi significativamente elevada ${ }^{4}$, enquanto outro estudo encontrou alta incidência em sessão única ${ }^{35}$. Paredes-Vieyra et al. ${ }^{36}$ (2012) afirmaram que os pacientes submetidos ao tratamento em sessão única apresentaram dor pós-operatória com menor frequência $(1,35 \%)$ do que aqueles em sessões múltiplas (2\%).

A eficácia do tratamento endodôntico em sessão única e em múltiplas sessões não é substancialmente diferente. A maioria das complicações é semelhante em termos de frequência, embora os pacientes submetidos à sessão única possam se deparar com um maior número de edema e estejam mais propensos a necessitar de medicação analgésica ${ }^{19}$. Sobre a reparação por meio da avaliação radiográfica revelou-se que o tratamento em sessão única parece ser um pouco mais eficaz do que em sessões múltiplas, mas não houve diferença estatisticamente significante ${ }^{19}$, resultados semelhantes foram obtidos por Sathorn et al. ${ }^{32}$ (2005) em sua revisão sistemática.

Um estudo que avaliou a eficácia e a dor pós-operatória em tratamentos endodônticos realizados em sessão única e sessões múltiplas não apresentou diferença estatisticamente significante na taxa de reparação e na dor pós-operatória ${ }^{19}$. No entanto, a maioria das revisões sistemáticas enfatiza principalmente a comparação de procedimentos sem considerar o estado pulpar pré-tratamento ${ }^{19,37}$.

Alguns estudos têm demonstrado a associação do estado pulpar e periapical com o sucesso do tratamento endodôntic ${ }^{5,33}$. No caso de polpa não vital, os canais estão geralmente infectados, especialmente na presença de periodontite apical. O controle efetivo da carga microbiana intracanal antes da obturação é um elemento-chave que conduz a uma elevada taxa de sucesso do tratamento ${ }^{8,33}$. Em polpas vitais, condições assépticas são mantidas após a instrumentação, e o foco principal do tratamento é prevenir a infecção iatrogênica do canal radicular. Consequentemente, a desinfecção dos canais, nesses casos, pode não ser necessária, em comparação 
com os casos de polpas infectadas ${ }^{38}$. Apesar disso, Imura e Zuolo ${ }^{24}$ (1995) não encontraram correlação entre vitalidade dos dentes e dor pós-operatória.

De acordo com os resultados da análise obtida no estudo de Su et al. ${ }^{29}$ (2011), a taxa de reparação após o tratamento endodôntico realizado em sessão única ou múltiplas foi semelhante para os dentes infectados. A prevalência de dor pós-obturação, em curto prazo, foi significativamente menor em sessão única. Destaca-se que ainda são necessários mais ensaios clínicos randomizados para elucidar esse questionamento.

A prevenção da dor pós-operatória pode ser obtida por meio da técnica crown-down, iniciando o pré-alargamento do terço cervical/médio e após apical, o que reduz o potencial de pressão hidrostática em direção apical e evita a passagem de material e restos teciduais para região periapical ${ }^{34}$. A sobreinstrumentação e a sobreobturação mostraram um aumento na dor pós-operatória ${ }^{38}$.

Após o tratamento endodôntico, os resultados só podem ser observados após um determinado período. A probabilidade de reparação periapical aumenta ao longo do tempo, e alguns pesquisadores sugeriram que seria necessário não menos do que quatro ou cinco anos após o tratamento para avaliar o processo de cura ${ }^{15,33}$.

\section{Considerações finais}

O sucesso e o fracasso do tratamento endodôntico em sessão única ou múltipla mostraram-se semelhantes, além de serem determinados por acompanhamento em longo prazo e não pela presença ou ausência de dor pós-operatória em curto prazo. Assim, a tomada de decisão clínica de optar por um tratamento em sessão única ou em múltiplas sessões deve ser baseada em evidências clínico-científicas, e não simplesmente em um consenso.

\section{Abstract}

Current technological advances and the improvement of chemo-mechanical preparation for cleaning and shaping the root canal have reduced the time required for this procedure, enabling treatment completion in one single visit. Objective: To discuss and compare through scientific evidence the findings on postoperative pain and healing rates in endodontic treatments performed in single and multiple visits. This literature review was based on a search for studies in PubMed, Science Direct, Scopus, Scielo, and Medline databases. Literature review: The single-visit endodontic treatment may be performed in cases diagnosed as irreversible pulpitis. However, in cases of pulp necrosis with or without apical periodontitis, the literature is controversial and opinions vary on the risks and benefits achieved in single or multiple visits. Along with other advantages, including time saving, cost-benefit, better patient complian- ce, and reduced risk of infection in-between sessions, the single visit may be suggested, but in some cases, as in the presence of exudate, it should not be recommended. Final considerations: Single- and multiple-visits treatment showed similar results considering healing rate and postoperative pain. Thus, the clinical decision-making for choosing an endodontic treatment in single or multiple visits should be based on clinical and scientific evidence.

Keywords: Endodontics. Postoperative pain. Treatment.

\section{Referências}

1. Salehrabi R, Rotstein I. Endodontics treatment outcomes in a large patient population in the USA: an epidemiological study. J Endod 2004; 30(12):846-50.

2. Imura N, Pinheiro ET, Gomes BP, Zaia AA, Ferraz CC, Souza-Filho FJ. The outcome of endodontic treatment: a retrospective study of 2000 cases performed by a specialist. J Endod 2007; 33(11):1278-82.

3. El Mubarak AH, Abu-bakr NH, Ibrahim YE. Postoperative pain inmultiple-visit and single-visit root canal treatment. J Endod 2010; 36(1):36-9.

4. Eleazer PD, Eleazer KR. Flare-up rate in pulpally necrotic molars in one-visit versus two-visit endodontic treatment. J Endod 1998; 24(9):614-6.

5. Albashaireh ZS, Alnegrish AS. Postobturation pain after single- and multiple-visit endodontic therapy. J Dent 1998; 26(3):227-32.

6. Abbott PV, Yu C. A clinical classification of the status of the pulp and the root canal system. Aust Dent J 2007; 52(1):17-31.

7. Byström A, Sundqvist G. Bacteriologic evaluation of the effect of 0.5 percent sodium hypochlorite in endodontic therapy. Oral Surg Oral Med Oral Pathol 1983; 55(3):307-12.

8. Sjögren U, Figdor D, Persson S, Sundqvist G. Influence of infection at the time of root filling on the outcome of endodontic treatment of teeth with apical periodontitis. Int Endod J 1997; 30(5):297-306.

9. Holland R, Soares IJ, Soares IM. Influence of irrigation and intracanal dressing on the healing process of dog's teeth with apical periodontitis. Endod Dent Traumatol 1992; 8(6):223-9.

10. Dodge JS. Immediate root filling. Dent Cosm 1887; 29:234-5.

11. Hofheinz RH. Immediate root filling. Dent Cosm 1892; 34:182-7.

12. Mohammadi Z, Farhad A, Tabrizizadeh M. One-visit versus multiple-visit endodontic therapy - a review. Int Dent J 2006; 56(5):289-93.

13. Orstavik D, Kerekes K, Molven O. Effects of extensive apical reaming and calcium hydroxide dressing on bacterial infection during treatment of apical periodontitis: a pilot study. Int Endod J 1991; 24(1):1-7.

14. Trope M, Delano EO, Orstavik D. Endodontic treatment of teeth with apical periodontitis: single vs. multivisit treatment. J Endod 1999; 25(5):345-50.

15. Weiger R, Rosendahl R, Löst C. Influence of calcium hydroxide intracanal dressings on the prognosis of teeth with endodontically induced periapical lesions. Int Endod J 2000; 33(3):219-26.

16. Oliet S. Single-visit endodontics: a clinical study. J Endod 1983; 9(4):147-52.

17. Gurgel-Filho ED, Vivacqua-Gomes N, Gomes BP, Ferraz $\mathrm{CC}$, Zaia AA, Souza-Filho FJ. In vitro evaluation of the ef- 
fectiveness of the chemomechanical preparation against $E n$ terococcus faecalis after single- or multiple-visit root canal treatment. Braz Oral Res 2007; 21(4):308-13.

18. Xavier AC, Martinho FC, Chung A, Oliveira LD, Jorge AO, Valera $\mathrm{MC}$ et al. One-visit versus two-visit root canal treatment: effectiveness in the removal of endotoxins and cultivable bacteria. J Endod 2013; 39(8):959-64.

19. Figini L, Lodi G, Gorni F, Gagliani M. Single versus multiple visits for endodontic treatment of permanent teeth: a Cochrane systematic review. J Endod 2008; 34(9):1041-7.

20. Wong AW, Zhang C, Chu CH. A systematic review of nonsurgical single-visit versus multiple-visit endodontic treatment. Clin Cosmet Investig Dent 2014; 6:45-56.

21. Sathorn C, Parashos P, Messer H. Australian endodontists' perceptions of single and multiple visit root canal treatment. Int Endod J 2009; 42(9):811-8.

22. Kvist T, Molander A, Dahlén G, Reit C. Microbiological evaluation of one- and two-visit endodontic treatment of teeth with apical periodontitis: a randomized, clinical trial. J En$\operatorname{dod} 2004 ; 30(8): 572-6$.

23. Jurcak JJ, Bellizzi R, Loushine RJ. Successful single-visit endodontics during Operation Desert Shield. J Endod 1993; 19(8):412-3

24. Imura N, Zuolo ML. Factors associated with endodontic flare-ups: a prospective study. Int Endod J 1995; 28(5):261-5.

25. Whitten BH, Gardiner DL, Jeansonne BG, Lemon RR. Current trends in endodontic treatment: report of a national survey. J Am Dent Assoc 1996; 127(9):1333-41.

26. Qualtrough AJ, Whitworth JM, Dummer PM. Preclinical endodontology: an international comparison. Int Endod J 1999; 32(5):406-14.

27. Carrotte P. 21st century endodontics: part 3. Int Dent J 2005; 55(4):247-53.

28. Yoldas O, Topuz A, Isçi AS, Oztunc H. Postoperative pain after endodontic retreatment: single- versus two-visit treatment. Oral Surg Oral Med Oral Pathol Oral Radiol Endod 2004; 98(4):483-7.

29. Su Y, Wang C, Ye L. Healing rate and post-obturation pain of single- versus multiple-visit endodontic treatment for infected root canals: a systematic review. J Endod 2011; 37(2):125-32.

30. Calhoun RL, Landers RR. One-appointment endodontic therapy: a nationwide survey of endodontists. J Endod 1982; 8(1):35-40.

31. Sjögren U, Hagglund B, Sundqvist G, Wing K. Factors affecting the long-term results of endodontic treatment. J Endod 1990; 16(10):498-504.

32. Sathorn C, Parashos P, Messer HH. Effectiveness of singleversus multiple-visit endodontic treatment of teeth with apical periodontitis: a systematic review and meta-analysis. Int Endod J 2005; 38(6):347-55

33. Peters LB, Wesselink PR. Periapical healing of endodontically treated teeth in one and two visits obturated in the presence or absence of detectable microorganisms. Int Endod J 2002; 35(8):660-7.

34. Siqueira Jr JF, Rôças IN, Favieri A, Machado AG, Gahyva SM, Oliveira JC et al. Incidence of postoperative pain after intracanal procedures based on an antimicrobial strategy. $\mathrm{J}$ Endod 2002; 28(6):457-60.

35. Oginni AO, Udoye CI. Endodontic flareups: comparison of incidence between single and multiple visit procedures in patients attending a Nigerian teaching hospital. BMC Oral Health 2004; 4(1):4

36. Paredes-Vieyra J, Enriquez FJ. Success rate of singleversus two-visit root canal treatment of teeth with apical periodontitis: a randomized controlled trial. J Endod 2012; 38(9):1164-9.
37. Sathorn C, Parashos P, Messer H. The prevalence of postoperative pain and flare-up in single- and multiple-visit endodontic treatment: a systematic review. Int Endod J 2008; 41(2):91-9.

38. Gesi A, Hakeberg M, Warfvinge J, Bergenholtz G. Incidence of periapical lesions and clinical symptoms after pulpectomy: a clinical and radiographic evaluation of 1- versus 2-session treatment. Oral Surg Oral Med Oral Pathol Oral Radiol Endod 2006; 101(3):379-88.

\section{Endereço para correspondência:}

Marcos Sergio Endo

Departamento de Odontologia

Universidade Estadual de Maringá

Avenida Mandacaru, 1.550, Bloco S08

87083-170 Maringá-PR

Telefone: (44) 9703-1672

E-mail: marcossendo@gmail.com

Recebido: 12/05/15. Aceito: 04/11/15. 